\title{
ON THE UNIVALENCE OF GENERAL INTEGRAL OPERATOR
}

\author{
B.A. Frasin, T. Al-Hawary, F. Yousef
}

Abstract. The object of the present paper is to obtain new univalence conditions for the general integral operator $I_{n}(z)=\left[\delta \int_{0}^{z} t^{\delta-1} \prod_{j=1}^{n}\left(\frac{f_{j}(t)}{g_{j}(t)}\right)^{\alpha_{j}}\left(\frac{f_{j}^{\prime}(t)}{g_{j}^{\prime}(t)}\right)^{\beta_{j}} d t\right]^{\frac{1}{\delta}}$ defined in the open unit $\operatorname{disc} \mathcal{U}=\{z:|z|<1\}$ where the functions $f_{j}, g_{j}$ are analytic in $\mathcal{U}, \alpha_{j}, \beta_{j}$ and $\delta$ are complex numbers.

2010 Mathematics Subject Classification: 30C45.

Keywords: Analytic and univalent functions, univalence condition, integral operator.

\section{Introduction AND DeFinitions}

Let $\mathcal{A}$ denote the class of all analytic functions $f$ in the open unit $\operatorname{disc} \mathcal{U}=\{z:|z|<1\}$ and normalized by the conditions $f(0)=f^{\prime}(0)-1=0$. Further, by $\mathcal{S}$ we shall denote the class of all functions in $\mathcal{A}$ which are univalent in $\mathcal{U}$.

A function $f(z) \in \mathcal{A}$ is said to be a member of the class $\mathcal{B}(\gamma)$ if it satisfies

$$
\left|\frac{z^{2} f^{\prime}(z)}{f^{2}(z)}-1\right|<1-\gamma
$$

for some $\gamma(0 \leq \gamma<1)$ and for all $z \in \mathcal{U}$. The class $\mathcal{B}(\gamma)$ was introduced and studied by Frasin and Darus [13].

Ponnusamy and Sing [20] studied the subclass $\mathcal{S}_{\mu}$ of analytic functions defined as follows

$$
\mathcal{S}_{\mu}=\left\{f \in \mathcal{A}:\left|\frac{z f^{\prime}(z)}{f(z)}-1\right|<\mu|z|, 0<\mu \leq 1, z \in \mathcal{U}\right\}
$$

The problem of finding sufficient conditions for univalence of various integral operators has been investigated in many recent works (see, for example, $[2,4,5,6$, $8,10,16,17,18])$. 
In [19] (see also [1]), Pescar obtained new univalence criteria for the integral operator defined by

$$
I_{n}(z)=\left[\delta \int_{0}^{z} t^{\delta-1} \prod_{j=1}^{n}\left(\frac{f_{j}(t)}{g_{j}(t)}\right)^{\alpha_{j}}\left(\frac{f_{j}^{\prime}(t)}{g_{j}^{\prime}(t)}\right)^{\beta_{j}} d t\right]^{\frac{1}{\delta}}
$$

where the functions $f_{j}, g_{j} \in \mathcal{A}, \alpha_{j}, \beta_{j} \in \mathbb{C} ; j=1, \ldots, n, n \in \mathbb{N}$ and $\delta \in \mathbb{C} \backslash\{0\}$. Here and throughout in the sequel every many-valued function is taken with the principal branch.

We observe that the above integral operator $I_{n}(z)$ generalizes some integral operators introduced by several researchers, for example.

1. If $g_{j}(z)=z ; j=1, \ldots, n$, then the integral operator $I_{n}(z)$ reduces to the integral operator

$$
F_{n}(z)=\left[\delta \int_{0}^{z} t^{\delta-1} \prod_{j=1}^{n}\left(\frac{f_{j}(t)}{t}\right)^{\alpha_{j}}\left(f_{j}^{\prime}(t)\right)^{\beta_{j}} d t\right]^{\frac{1}{\delta}},
$$

introduced by Frasin [12] (see also [11]).

2. If $\beta_{j}=0 ; j=1, \ldots, n$, then the integral operator $I_{n}(z)$ reduces to the integral operator

$$
G_{n}(z)=\left[\delta \int_{0}^{z} t^{\delta-1} \prod_{j=1}^{n}\left(\frac{f_{j}(t)}{g_{j}(t)}\right)^{\alpha_{j}} d t\right]^{\frac{1}{\delta}},
$$

introduced by Moldoveanu et al. [14].

3. If $g_{j}(z)=z$ and $\beta_{j}=0 ; j=1, \ldots, n$, then the integral operator $I_{n}(z)$ reduces to the integral operator

$$
D_{n}(z)=\left[\delta \int_{0}^{z} t^{\delta-1} \prod_{j=1}^{n}\left(\frac{f_{j}(t)}{t}\right)^{\alpha_{j}} d t\right]^{\frac{1}{\delta}}
$$

introduced by Breaz and Breaz [3]. 
In our present investigation, we study some univalence conditions for the integral operator $I_{n}(z)$ if the analytic functions $f_{j}(z)$ and $g_{j}(z)$ are in the classes $\mathcal{B}\left(\gamma_{j}\right)$ and $\mathcal{S}_{\mu_{j}} ; j=1, \ldots, n$.

In order to derive our main results, we need the following lemmas.

Lemma 1. ([n]) If $f(z) \in \mathcal{B}(\gamma)$, then

$$
\left|\frac{z f^{\prime}(z)}{f(z)}-1\right|<\frac{(1-\gamma)(1+|z|)}{1-|z|} \quad(0 \leq \gamma<1, z \in \mathcal{U}) .
$$

Lemma 2. ([9]) If $f(z) \in \mathcal{B}(\gamma)$, then

$$
\left|\frac{z f^{\prime \prime}(z)}{f^{\prime}(z)}\right|<\frac{(1-\gamma)(2+|z|)}{1-|z|} \quad(0 \leq \gamma<1, z \in \mathcal{U})
$$

Lemma 3. ([15]) Let $\lambda \in \mathbb{C}$ with $\operatorname{Re}(\lambda)>0$. If $k \in \mathcal{A}$ satisfies

$$
\frac{1-|z|^{2 \operatorname{Re}(\lambda)}}{\operatorname{Re}(\lambda)}\left|\frac{z k^{\prime \prime}(z)}{k^{\prime}(z)}\right| \leq 1,
$$

for all $z \in \mathcal{U}$, then, for any complex number $\zeta$, with $\operatorname{Re}(\zeta) \geq \operatorname{Re}(\lambda)$, the integral operator

$$
F_{\zeta}(z)=\left\{\zeta \int_{0}^{z} t^{\zeta-1} k^{\prime}(t) d t\right\}^{\frac{1}{\zeta}}
$$

is in the class $\mathcal{S}$.

\section{Univalence Conditions for the Integral Operator $I_{n}$}

First, we give univalence conditions for the integral operator $I_{n}(z)$ where the analytic functions $f_{j}(z)$ and $g_{j}(z)$ are in the class $\mathcal{B}\left(\gamma_{j}\right) ; j=1, \ldots, n$.

Theorem 4. Let the analytic functions $f_{j}(z)$ and $g_{j}(z)$ be in the class $\mathcal{B}\left(\gamma_{j}\right) ; 0 \leq$ $\gamma_{j}<1 ; j=1, \ldots, n$ and satisfy the inequality

$$
\sum_{j=1}^{n}\left(1-\gamma_{j}\right)\left(4\left|\alpha_{j}\right|+6\left|\beta_{j}\right|\right) \leq\left\{\begin{array}{c}
a, \text { if } 0<a<\frac{1}{2} \\
\frac{1}{2}, \text { if } \frac{1}{2}<a<\infty
\end{array}\right.
$$

where $\lambda \in \mathbb{C}$ with $\operatorname{Re}(\lambda)=a>0$, then the integral operator $I_{n}(z)$ defined by (3) is analytic and univalent in $\mathcal{U}$. 
Proof. Define the regular function $H(z)$ by

$$
H(z)=\int_{0}^{z}\left(\frac{f_{1}(t)}{g_{1}(t)}\right)^{\alpha_{1}} \cdots\left(\frac{f_{n}(t)}{g_{n}(t)}\right)^{\alpha_{n}}\left(\frac{f_{1}^{\prime}(t)}{g_{1}^{\prime}(t)}\right)^{\beta_{1}} \cdots\left(\frac{f_{n}^{\prime}(t)}{g_{n}^{\prime}(t)}\right)^{\beta_{n}} d t .
$$

Clearly $H \in \mathcal{A}$, i.e. $H(0)=H^{\prime}(0)-1=0$. On the other hand, it is easy to see that

$$
\frac{z H^{\prime \prime}(z)}{H^{\prime}(z)}=\sum_{j=1}^{n} \alpha_{j}\left[\left(\frac{z f_{j}^{\prime}(t)}{f_{j}(t)}-\frac{z g_{j}^{\prime}(t)}{g_{j}(t)}\right)+\beta_{j}\left(\frac{z f_{j}^{\prime \prime}(t)}{f_{j}^{\prime}(t)}-\frac{z g_{j}^{\prime \prime}(t)}{g_{j}^{\prime}(t)}\right)\right]
$$

or, equivalently,

$$
\frac{z H^{\prime \prime}(z)}{H^{\prime}(z)}=\sum_{j=1}^{n}\left\{\alpha_{j}\left[\left(\frac{z f_{j}^{\prime}(t)}{f_{j}(t)}-1\right)-\left(\frac{z g_{j}^{\prime}(t)}{g_{j}(t)}-1\right)\right]+\beta_{j}\left(\frac{z f_{j}^{\prime \prime}(t)}{f_{j}^{\prime}(t)}-\frac{z g_{j}^{\prime \prime}(t)}{g_{j}^{\prime}(t)}\right)\right\} .
$$

Since the analytic functions $f_{j}(z)$ and $g_{j}(z)$ are in the class $\mathcal{B}\left(\gamma_{j}\right) ; j=1, \ldots, n$, from (7), (8) and (12), we obtain

$$
\begin{aligned}
\left|\frac{z H^{\prime \prime}(z)}{H^{\prime}(z)}\right| \leq & \sum_{j=1}^{n}\left\{\left|\alpha_{j}\right|\left[\left|\frac{z f_{j}^{\prime}(t)}{f_{j}(t)}-1\right|+\left|\frac{z g_{j}^{\prime}(t)}{g_{j}(t)}-1\right|\right]\right. \\
& \left.+\left|\beta_{j}\right|\left[\left|\frac{z f_{j}^{\prime \prime}(t)}{f_{j}^{\prime}(t)}\right|+\left|\frac{z g_{j}^{\prime \prime}(t)}{g_{j}^{\prime}(t)}\right|\right]\right\} \\
\leq & \sum_{j=1}^{n}\left\{2\left(1-\gamma_{j}\right)\left|\alpha_{j}\right|\left(\frac{1+|z|}{1-|z|}\right)+2\left(1-\gamma_{j}\right)\left|\beta_{j}\right|\left(\frac{2+|z|}{1-|z|}\right)\right\} \\
\leq & \frac{1}{1-|z|} \sum_{j=1}^{n}\left(1-\gamma_{j}\right)\left(4\left|\alpha_{j}\right|+6\left|\beta_{j}\right|\right),
\end{aligned}
$$

for all $z \in \mathcal{U}$. Multiply both sides of $(13)$ by $\frac{1-|z|^{2} \operatorname{Re}(\lambda)}{\operatorname{Re}(\lambda)}$, we get

$$
\frac{1-|z|^{2 \operatorname{Re}(\lambda)}}{\operatorname{Re}(\lambda)}\left|\frac{z H^{\prime \prime}(z)}{H^{\prime}(z)}\right| \leq \frac{1-|z|^{2 \operatorname{Re}(\lambda)}}{(\operatorname{Re}(\lambda))(1-|z|)} \sum_{j=1}^{n}\left(1-\gamma_{j}\right)\left(4\left|\alpha_{j}\right|+6\left|\beta_{j}\right|\right)
$$

for all $z \in \mathcal{U}$.

Define the function $\Phi(x)=\frac{1-x^{2 a}}{1-x}$, where $|z|=x, x \in[0,1)$, and $\operatorname{Re}(\lambda)=a>0$. It is easy to prove that

$$
\Phi(x) \leq\left\{\begin{aligned}
1, & \text { if } 0<a<\frac{1}{2} \\
2 a, & \text { if } \frac{1}{2}<a<\infty .
\end{aligned}\right.
$$


From (14), (15) and the hypothesis (10), we have

$$
\begin{aligned}
\frac{1-|z|^{2 a}}{a}\left|\frac{z H^{\prime \prime}(z)}{H^{\prime}(z)}\right| & \leq\left\{\begin{array}{c}
\frac{1}{a} \sum_{j=1}^{n}\left(1-\gamma_{j}\right)\left(4\left|\alpha_{j}\right|+6\left|\beta_{j}\right|\right), \text { if } 0<a<\frac{1}{2} \\
2 \sum_{j=1}^{n}\left(1-\gamma_{j}\right)\left(4\left|\alpha_{j}\right|+6\left|\beta_{j}\right|\right), \text { if } \frac{1}{2}<a<\infty
\end{array}\right. \\
& \leq 1,
\end{aligned}
$$

for all $z \in \mathcal{U}$. Applying Lemma 3 for the function $H(z)$, we prove that $I_{n}(z) \in \mathcal{S}$. Thus, the proof is complete

Our next result gives univalence conditions for the integral operator $I_{n}(z)$ where the analytic functions $f_{j}(z)$ and $g_{j}(z)$ are in the class $\mathcal{S}_{\mu_{j}} ; j=1, \ldots, n$.

Theorem 5. Let the analytic functions $f_{j}(z)$ and $g_{j}(z)$ be in the class $\mathcal{S}_{\mu_{j}} ; 0<$ $\mu_{j} \leq 1 ; j=1, \ldots, n$ and satisfy the inequality

$$
\left|\frac{z f_{j}^{\prime \prime}(z)}{f_{j}^{\prime}(z)}-\frac{z g_{j}^{\prime \prime}(z)}{g_{j}^{\prime}(z)}\right|<|z| \quad(j=1, \ldots, n, z \in \mathcal{U}) .
$$

If $\lambda \in \mathbb{C}$ with $\operatorname{Re}(\lambda)=a>0$ and

$$
\sum_{j=1}^{n}\left(2 \mu_{j}\left|\alpha_{j}\right|+\beta_{j}\right) \leq \frac{(2 a+1)^{\frac{2 a+1}{2 a}}}{2},
$$

then the integral operator $I_{n}(z)$ defined by (3) is analytic and univalent in $\mathcal{U}$.

Proof. Let the analytic functions $f_{j}(z)$ and $g_{j}(z)$ be in the class $\mathcal{S}_{\mu_{j}} ; 0<\mu_{j} \leq 1$; $j=1, \ldots, n$. Then from (2) and (12) we get

$$
\begin{aligned}
\left|\frac{z H^{\prime \prime}(z)}{H^{\prime}(z)}\right| \leq & \sum_{j=1}^{n}\left\{\left|\alpha_{j}\right|\left[\left|\frac{z f_{j}^{\prime}(t)}{f_{j}(t)}-1\right|+\left|\frac{z g_{j}^{\prime}(t)}{g_{j}(t)}-1\right|\right]\right. \\
& \left.+\left|\beta_{j}\right|\left|\frac{z f_{j}^{\prime \prime}(t)}{f_{j}^{\prime}(t)}-\frac{z g_{j}^{\prime \prime}(t)}{g_{j}^{\prime}(t)}\right|\right\} \\
\leq & \sum_{j=1}^{n}\left(2 \mu_{j}\left|\alpha_{j}\right|+\beta_{j}\right)|z| .
\end{aligned}
$$

Thus, we have

$$
\frac{1-|z|^{2 a}}{a}\left|\frac{z H^{\prime \prime}(z)}{H^{\prime}(z)}\right| \leq \frac{1}{a} \sum_{j=1}^{n}\left(2 \mu_{j}\left|\alpha_{j}\right|+\beta_{j}\right)|z|\left(1-|z|^{2 a}\right) \quad(z \in \mathcal{U}) .
$$


Let us denote $|z|=x, x \in[0,1], \operatorname{Re}(\lambda)=a>0$ and $\Psi(x)=x\left(1-x^{2 a}\right)$. It is easy to prove that the maximum is attained at the point $x=1 /(2 a+1)^{1 / 2 a}$ and therefore we have

$$
\Psi(x) \leq \frac{2 a}{(2 a+1)^{\frac{2 a+1}{2 a}}} .
$$

In view of this inequality and (17), we obtain

$$
\frac{1-|z|^{2 a}}{a}\left|\frac{z H^{\prime \prime}(z)}{H^{\prime}(z)}\right| \leq 1 \quad(z \in \mathcal{U}) .
$$

Applying Lemma 3 for the function $H(z)$, we prove that $I_{n}(z) \in \mathcal{S}$.

We conclude our present investigation by mentioning that, by suitably specializing the parameters involved, our main results would yield the following new univalence conditions for some integral operators introduced in Section 1.

Corollary 6. Let the analytic functions $f_{j}(z)$ in the class $\mathcal{B}\left(\gamma_{j}\right) ; 0 \leq \gamma_{j}<1$; $j=1, \ldots, n$ and satisfy the inequality

$$
\sum_{j=1}^{n}\left(1-\gamma_{j}\right)\left(4\left|\alpha_{j}\right|+6\left|\beta_{j}\right|\right) \leq\left\{\begin{array}{cc}
a, & \text { if } 0<a<\frac{1}{2} \\
\frac{1}{2}, & \text { if } \frac{1}{2}<a<\infty
\end{array}\right.
$$

where $\lambda \in \mathbb{C}$ with $\operatorname{Re}(\lambda)=a>0$, then the integral operator $F_{n}(z)$ defined by (4) is analytic and univalent in $\mathcal{U}$.

Corollary 7. Let the analytic functions $f_{j}(z)$ in the class $\mathcal{B}\left(\gamma_{j}\right) ; 0 \leq \gamma_{j}<1$; $j=1, \ldots, n$ and satisfy the inequality

$$
\sum_{j=1}^{n}\left(1-\gamma_{j}\right)\left|\alpha_{j}\right| \leq\left\{\begin{array}{c}
\frac{a}{4}, \text { if } 0<a<\frac{1}{2} \\
\frac{1}{8}, \text { if } \frac{1}{2}<a<\infty
\end{array}\right.
$$

where $\lambda \in \mathbb{C}$ with $\operatorname{Re}(\lambda)=a>0$, then the integral operator $G_{n}(z)$ defined by (5) is analytic and univalent in $\mathcal{U}$.

Corollary 8. Let the analytic functions $f_{j}(z)$ be in the class $\mathcal{S}_{\mu_{j}} ; 0<\mu_{j} \leq 1$; $j=1, \ldots, n$ and satisfy the inequality

$$
\left|\frac{z f_{j}^{\prime \prime}(z)}{f_{j}^{\prime}(z)}\right|<|z| \quad(j=1, \ldots, n, z \in \mathcal{U}) .
$$




$$
\begin{aligned}
& \text { If } \lambda \in \mathbb{C} \text { with } \operatorname{Re}(\lambda)=a>0 \text { and } \\
& \qquad \sum_{j=1}^{n}\left(2 \mu_{j}\left|\alpha_{j}\right|+\beta_{j}\right) \leq \frac{(2 a+1)^{\frac{2 a+1}{2 a}}}{2},
\end{aligned}
$$

then the integral operator $F_{n}(z)$ defined by (4) is analytic and univalent in $\mathcal{U}$.

Corollary 9. Let the analytic functions $f_{j}(z)$ and $g_{j}(z)$ be in the class $\mathcal{S}_{\mu_{j}} ; 0<$ $\mu_{j} \leq 1 ; j=1, \ldots, n$ and satisfy the inequality

$$
\left|\frac{z f_{j}^{\prime \prime}(z)}{f_{j}^{\prime}(z)}-\frac{z g_{j}^{\prime \prime}(z)}{g_{j}^{\prime}(z)}\right|<|z| \quad(j=1, \ldots, n, z \in \mathcal{U}) .
$$

If $\lambda \in \mathbb{C}$ with $\operatorname{Re}(\lambda)=a>0$ and

$$
\sum_{j=1}^{n} \mu_{j}\left|\alpha_{j}\right| \leq \frac{(2 a+1)^{\frac{2 a+1}{2 a}}}{4}
$$

then the integral operator $D_{n}(z)$ defined by (6) is analytic and univalent in $\mathcal{U}$.

\section{REFERENCES}

[1] C. L. Aldea and V. Pescar, Univalence criteria for a general integral operator, Bulletin of the Transilvania University of Braşov 10, 59 (2017), 19-30.

[2] D. Blezu and R.N. Pascu, Univalence criteria for integral operators, Glasnik Math. 36, 56 (2001), 241-245.

[3] D. Breaz and N. Breaz, Two Integral Operators, Studia Univ. Babeş-Bolyai Math. 47, 3 (2002), 13-21.

[4] D. Breaz and N. Breaz, Univalence conditions for certain integral operators, Studia Univ. Babeş-Bolyai Math., Vol. XLVII No.2 (2002), 9-15.

[5] D. Breaz and V. Pescar, Univalence conditions for some general integral operators, Banach J. Math. Anal. 1, 2 (2008), 53-58.

[6] S. Bulut, Uninvalence preserving integral operators defined by generalized AlOboudi differential operators, An. Şt. Univ. Ovidius Constanţa, 17, 1 (2009), 37-50.

[7] E. Deniz and H. Orhan, An extension of the univalence criterion for a family of integral operators, Ann. Univ. Mariae Curie-Skłodowska Sect. A 64, 2 (2010), 29-35.

[8] B.A. Frasin, New criteria for univalence of certain integral operators, Acta Mathematica Academiae Paedagogicae Nyiregyhaziensis, 27 (2011), 31-39. 
B.A. Frasin, T. Al-Hawary, F. Yousef - On the univalence of ...

[9] B. A. Frasin, A note on certain analytic and univalent functions, Southeast Asian J. Math. 28 (2004), 829-836.

[10] B.A. Frasin, Univalence of two general integral operator, Filomat, Vol. 23, 3, (2009), 223-229.

[11] B.A. Frasin, New general integral operator, Comp. Math. Appl. 62 (2011), $4272-$ 4276 .

[12] B.A. Frasin, Order of convexity and univalence of general integral operator, Journal of the Franklin Institute, 348 (2011), 1013-1019.

[13] B. A. Frasin and M. Darus, On certain analytic univalent functions, Internat. J. Math. and Math. Sci.25 (5) (2001), 305-310.

[14] S. Moldoveanu, H. Ovesea and N. N. Pascu, On the univalence of an integral operator, Seminar of Geometric Function Theory, Bra sov, Preprint Nr. 2 (1991), 63-66.

[15] N. Pascu, An improvement of Backer's univalence criterion, Proceedings of the Commemorative Session Simion Stoilow, Brasov, (1987), 43-48.

[16] N. Pascu and V. Pescar, On the integral operators of Kim-Merkis and Pfaltzgraff, Mathematica, 32 (55), 2(1990), 185-192.

[17] V. Pescar, Univalence of certain integral operators, Acta Univ. Apul. 12 (2006), 43-48.

[18] V. Pescar, On some integral classes of integral operators, General Math. 16, 1 (2008), 11-17.

[19] V. Pescar, New univalence criteria for some integral operators, Studia Univ. Babeş-Bolyai Math. 59, 2 (2014), 167-176.

[20] S. Ponnusamy and V. Singh, Critreria for univalent, starlike and convex functions, Bull. Belg. Math. Soc. 9 (2002), 511-531.

B.A. Frasin

Department of Mathematics, Faculty of Science,

Al al-Bayt University,

Mafraq, Jordan

email:bafrasin@yahoo.com

Tariq Al-Hawary

Department of Applied Science, Ajloun College,

Al-Balqa Applied University,

Ajloun 26816, Jordan

email: tariq_amh@yahoo.com 
B.A. Frasin, T. Al-Hawary, F. Yousef - On the univalence of ...

Feras Yousef

Department of Mathematics, Faculty of Science, University of Jordan,

Amman 11942, Jordan

email: fyousef@ju.edu.jo 